\title{
Compte rendu d'un double colloque sur l'étude des efforts des lames
}

\author{
An account of two meetings \\ on the study of wave loads
}

\author{
PAR A. DE ROUVILLE,
}

\author{
INSPECTEUT GŔNÉRAT DES PONTS ET CHAUSSEES ER. \\ FROFESSEUR HONORAIRI A L'ECOLE DES PONTS ET CHAUSSEES \\ PRÉSIDENT DE LA SECTION D'HYDRAULIOUE FIUVIALE ET MARTTIME DE LA S.M.F.
}

\begin{abstract}
Deux réunions récentes ont eu pour objet essentiel l'étude des efforts des lames, subsidiairement celles des seiches et des amarage: corrélatifs de navires, plus spécialement l'cxposé d'expériences faites en France (SO.GR.E. A.H.) sur les talus de digues soumises aux vagues déferlantes, ainsi que des réflexions concernant laction de la mer sur les digues verticales et sur les navires (M. Gille).

Ces réunions se sont tenaes respectivement en marge du $X^{\circ}$ Congres International de Navigation, à Baltinore (E.U.A., septembre 1961) où des communications étrangères ont été équlement exposées, et au titre de la Section d'Hydraulique fluviale et maritime de la S.H.F. (30 nonembre) plus particulièrement orientée. vers les travaux français de l'année 1961.
\end{abstract}

Ce fut d'abord le Comité pour l'étude des efforts dus aux lames et aux seiches relevant de l'A.I.P.C.N.

En Amérique, le Comité a tenu quatre séances, dont trois à Baltimore et la dernière à Washington, au Laboratoire du « Beach Erosion Board .

Peu de membres européens du Comité avaient pu se joindre aux Américains, forcément plus nombreux.

Deux documents avaient été imprimés à Bruxelles, au siège de l'Assnciation Internationale des Congrès de Navigation, et distribués par avance.

\begin{abstract}
The main subject considered in two recent meetings was the study of wave loads, including the forces affecting ships' moorings under seiche conditions, with a special exposé of experimental mork carried oul by SO.GR.E.A.H. in France on rubble mound embankments subjected to breaking wave action, and comments on sea action on pertical breakwaters and ships (M. Gille).

The subject of these meetings was associated with that of the Twentieth International $\mathrm{Na}$ vigation Congress at Baltimore (September 1961), were papers from foreign contributors were also read, and they were held in connection with the Meeting of the River and Maritime Hydraulics Section of "Sociéte $\mathrm{Hy}$ drotechnique de France" (30th November) which was more specifically concerned with work done in France in 1961.
\end{abstract}

Il s'agissait :

- (A) des activités du Comité International chargé de l'étude des efforts dus aux lames depuis l'origine;

- (B) d'une bibliographie encore incomplète, ou imparfaite en ce qu'elle ne contient aucune analyse des documents signalés et qu'elle est peut-être trop abondante.

Quelques collègues étrangers (Hollandais et Italiens) avaient produit des communications sur l'action des houlcs en eau peu profonde, d'une part, et sur les nouveaux résultats obte- 
nus aux stations dynomaréomotrices et stéréophotogrammétriques de Naples, d'autre part.

L'étude des Hollandais décrit les méthodes d'enregistrement des spectres de houles, les applications de la théorie de la force des lames aux portes d'écluses verticales et aux digues en pente douce. Elle indique comment l'action des lames sur les fonds mobiles a été étudiée par traceurs radioactifs. La note italienne expose les réflexions engendrées par les installations dynomaréomotrices, les corrections qui leur ont été apportées et les raisons des délauts de fonctionnement antérieurs, les modifications des installations qui permettent d'enregisirer en tout temps les efforts recus sur le mòle duc des Abruzzes; elle relate, enfin, en courbes et calculs, les effets d'une tempête assez forte de décembre 1960 , et rapproche les pressions théoriques calculées et observées.

$$
\text { * }
$$

Nous avous présenté ensuite, personnellement, les travaux français, exécutés spćcialement par la SO.GR.E.A.H. et constituant une étude sur modèle réduit des efforts des lames sur les talus des digues ou d'ouvrages de défense littorale.

Cet exposé, ainsi que les différentes communications ci-dessus, ont été repris pour la séance de la Commission d'Hydraulique fluviale et maritime du 30 novemhre 1961, où ils ont fait l'objel d'une discussion plus approfondie.

Nous donnons ci-dessous les principaux éléments de ce double exposé.

Il était expliqué en préambule que l'action sur les ouvrages verticaux avait paru déjà suffisamment avancée, que des études portant sur un lalus naturel de digue très irrégulier, encore insuffisamment élucidées, ne pouvaient être maintenant poursuivies, moyennant des dépenses raisonnables, que sur modèle.

\section{i}

Le canal à houle utilisé à Grenoble a une longueur de $27 \mathrm{~m}$, une largeur de $0,30 \mathrm{~m}$.

Les batteurs, pour houle régulière ou irrégulière, sont accompagnés de guideaux et de filtres régularisant les ondes et absorbant les réflexions. La plaque, figurant un talus de digue lisse ou un ouvrage de défense littorale, est une tôle rendue bien rigide et inclinable à volonté depuis $4 / 3$ jusqu'à $5 / 1$, en passant par $5 / 3,2 / 1$, $5 / 2,10 / 3$, c'est-à-dire depuis les talus protégeables avantageusement avec des tétrapodes, jusqu'aux cuirassements des ouvrages littoraux des Flandres.

Dix-sept capteurs sont échelonnés sur la génératrice médiane à des distances de $7 \mathrm{~cm}$.

Les capteurs sont à membranes, dont les dé- placements sont détectés électriquement et amplifiés par des appareils électroniques très sensibles, réalisés et mis au point à Grenoble même.

L'enregistrement se fait par spots lumineux impressionnant un papier, pour éviter l'amortissement par le frottement d'un style.

On mesure la composante de pression normale au parement; c'est celle qui met surtout en cause la stabilité d'une carapace lisse (par exemple couche bitumineuse).

On a travaillé uniquement avec des houles de cambrure-limite, c'est-à-dire déferlant sur le talus, pour être certain d'enregistrer des maximums; c'est le cas le plus menacant pour les digues à talus qu'assaillent généralement des lames en profondeur finie, et l'effet de poinçonnement est alors prépondérant

Pour chaque pente, on a fait varier la période ct, pour chaque période, on a ajusté l'amplitude qui donnait la cambrure-limite.

On a utilisé la variable réduite $h / L$ (profondeur relative) pour généraliser le problème, de facon que les essais soient valables quelles que soient les profondeurs dans la nature. Pour une profondeur et une longueur d'onde données en nature, quelles qu'elles soient, il existe un rapport $h / \mathrm{L}$ qui a été représenté en modèle.

Seize périodes ont été choisies, s'échelonnant de $0,65 \mathrm{~s}$ à $1,96 \mathrm{~s}$, ce qui permet bien de tracer une courbe et correspondrait, à l'échelle de $1 / 50$ par exemple $(h=15 \mathrm{~m})$, à des périodes réelles comprises entre $4,5 \mathrm{~s}$ et $14 \mathrm{~s}$.

On a utilisé comme variables, pour les courbes représentatives des phénomènes, les rapports sans effet d'échelle :

$h / \mathrm{L}$, profondeur relative;

$\mathrm{P}_{\mathrm{M}} / \mathrm{L}$, valeur, rapportée à la longueur d'onde, du maximum de pression enregistré sur le parement;

$x / h$, valeur, rapportée à la profondeur, de la position où se situe ce maximum.

Pour faciliter la transposition des résultats du modèle aux valeurs de la nature, on a fait abstraction de certaines caractéristiques, comme la cambrure $2 a / L_{\text {, }}$ puisqu'on a toujours travaillé en cambrure-limite, c'est-à-dire dans les conditions qui donnent chaque fois le maximum de pression à craindre, pour une profondeur, une longueur d'onde et une pente données.

\section{Cas de la houle régulière. Résultats.}

Les résultats ont été présentés sous la forme de 18 courbes, 6 photographies de déferlements en modèles, et un tableau sommaire.

Les 6 piemières courbes, qui sont doubles, figurent, pour chacune des 6 pentes de talus étudiées : 
-. A la partie supéricure, la variation du rapport $\mathrm{P}_{\mathrm{M}} / \mathrm{L}$ en fonction de la hauteur d'eau relative $h / L$;

- A la partie inférieure, et en fonction de la même abscisse $h / \mathrm{I}$, la variation du rapport $x / h$. On s'aperçoit que la cote du point d'impact maximal est toujours au-dessous du plan de repos.

L'allure du phénomène varie suivant la raideur de la pente du talus.

Pour les talus raides (voir fig. 1) (1), la pression monte en flèche très brusque, le maximum étant atteint pour une valeur précise et étroite de $h / \mathrm{L}$. On assiste à un véritable effet de gifle.

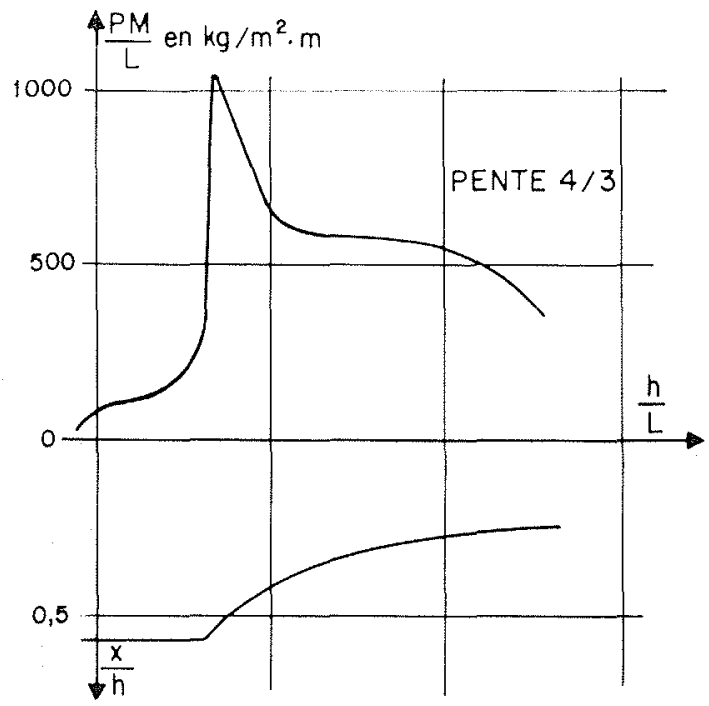

FIG. 1

Celte flèche tombe dès que la profondeur relative s'écarte un peu de ce rapport critique :

$(h / \mathrm{L}=0,16$ pour un talus à la pente $4 / 3)$.

Quant à la cote relative du point d'impact maximal, elle se place sur des courbes semblables, avec un ressaut plus accusé pour les forts talus, et naissant à peu près sous l'abscisse de la flèche précitée. Pour les grandes profondeurs relatives, elle est assez près du niveau de repos et varie peu avec $h / L$; mais elle s'abaisse quand la vague se creuse, que le rouleau se forme, qu'on arrive aux valeurs de $h / \mathrm{L}$ plus faibles, déterminantes d'ailleurs, des maximums de pres-

(1) Cette numérotation des figures est indépendante de celle, plus développée, du document R.8.106 de la SO.GR.E.A.H. (décembre 1961), non joint au présent compte rendu

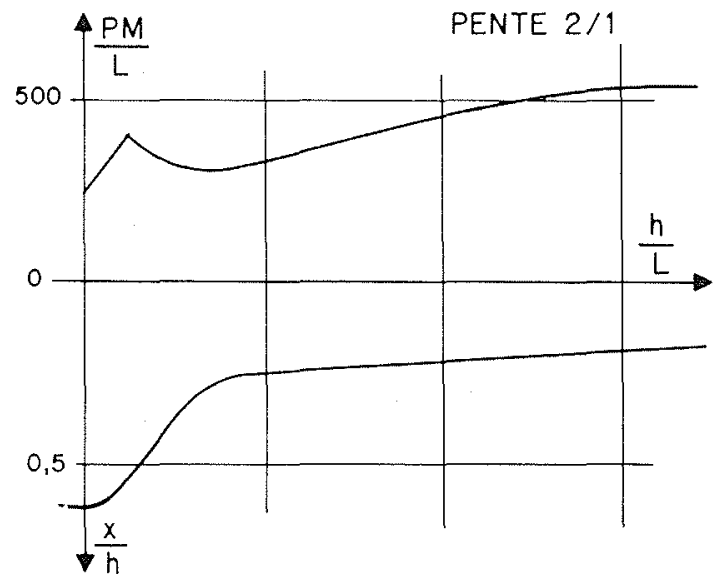

FIG. 2

sion. Cette cote se maintient basse ensuite pour les abscisses décroissantes de $h / L$.

Pour le talus d̀ pente moyenne, la pointe est à peine marquée; quoique s'esquissant, le phénomène de giffe est moins important, et réalisé pour un intervalle de $h / L$ relativement peu étroit (voir fig. 2, correspondant à la planche 3 du document 8.106).

Pour le talus de pente douce, le point est remplacé par une colline aplatie et étalée sur un intervalle assez grand de $h / L$ (voir fig. 3, correspondant à la planche 6 du document 8.106).

S'il y a quelques anomalies perceptibles, c'est, en dehors de l'hypothèse d'incertitudes expérimentales, le fait que le rouleau de déferlement attaque plus ou moins nettement le talus selon la pente et la période, que le jet de rive qu'il envoie lui coupe le pied et l'amortit sur les talus les plus faibles, esquissant même à l'œil un double impact à deux niveaux différents. Cela fait songer aux rouleaux successifs sur les plages à barre.

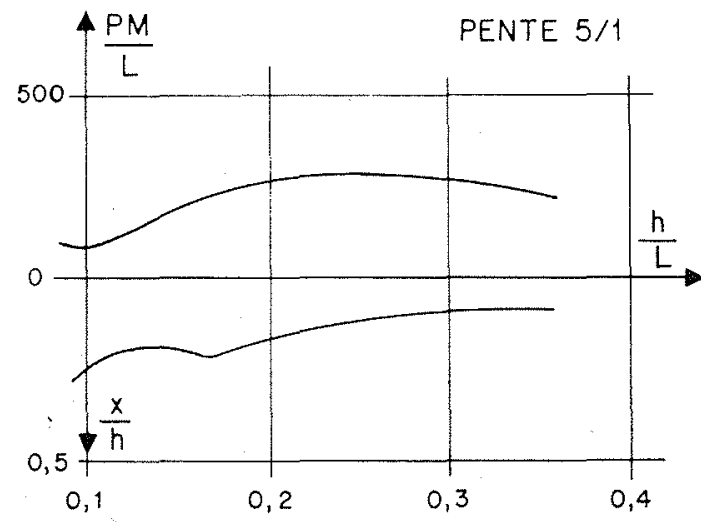

FIG, 3 


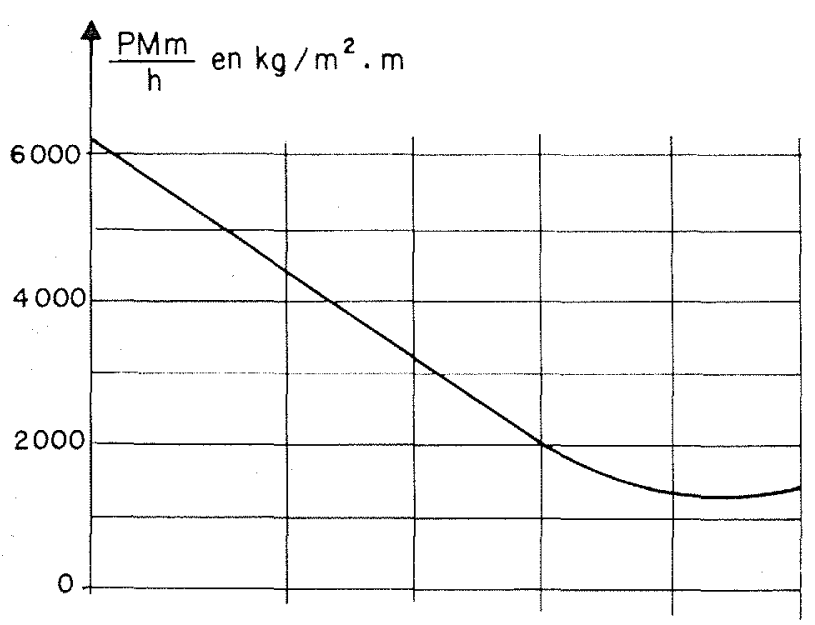

Fig. 4

La planche 7 du document 8.106 ou la figure 4 synthétise la variation de la pression «maximum maximorum » en fonction de la pente à talus; il suffit de multiplier l'ordonnée par la profondeur d'eau réelle pour avoir la pression maximale en $\mathrm{kg} / \mathrm{m}^{2}$.

On y lit tout de suite combien cette pression maxinale diminue avec la pente du talus, demeurant pratiquement étale à partir du talus $10 / 3$, celle que nous préconisons pour les talus des digues; elle tombe dans la proportion de 185 à 42 , soit $4,4 / 1$.

Un tableau donne, sous une autre forme, les pressions réelles variant :

de $12,6 \mathrm{t}$ à $56,5 \mathrm{t}$ par $\mathrm{m}^{2}$ pour des fonds de $(-9,00)$ (échelle $1 / 30)$;

de $21 \mathrm{t}$ à $92,5 \mathrm{t}$ pour des fonds de $(--15,00)$ (échelle 1/50);

de $33,6 \mathrm{t}$ à $148 \mathrm{t}$ pour des fonds de $(-24,00)$ (échelle 1/80).

La planche 8 du document 8.106 , reproduite (fig. 5), représente les valeurs de $h / L$ donnant la

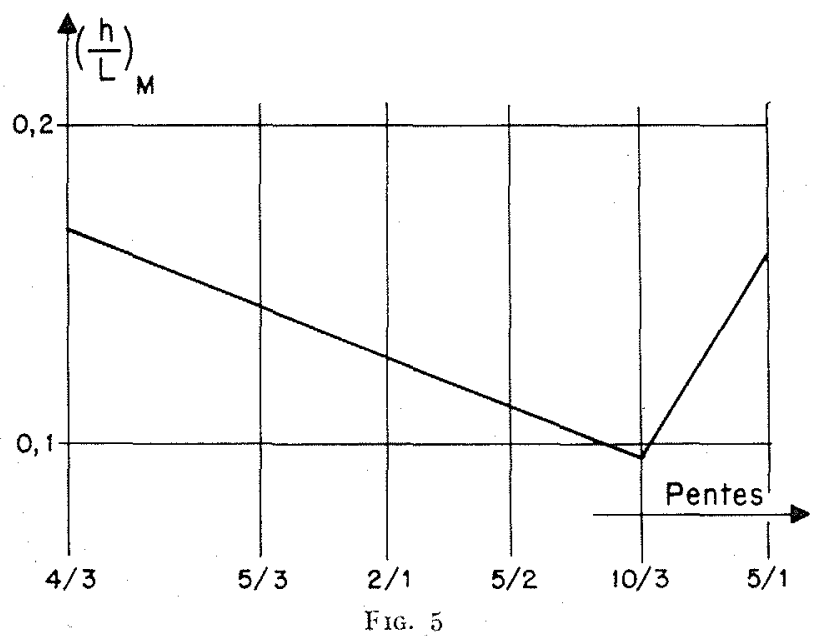

pression maximale pour les différents talus, c'est-à-dire la période de la houle la plus dangereuse pour le talus considéré (en se reportant à un abaque donnant la période d'après la longueur d'onde).

Elle révèle, par cette courbe en V, que les courtes longueurs d'onde ( $h / L$ grand) sont plus mauvaises pour les talus raides, tandis que les plus grandes longueurs d'onde sont plus mauvaises pour les lalus très doux.

Il intervient à la fois un effet de réflexion plus accentué sur les talus raides et un effet de freinage des ondes longues dans les faibles profondeurs d'eau où elles se propagent, ainsi qu'un effet de vitesse de reflux de la lame sur le parement. Cette influence $d u$ fond oblige, pour replacer le déferlement sur l'obstacle, à réduire l'amplitude de la houle au-dessous de celle qui correspondrait à la cambrure-limite en profon-

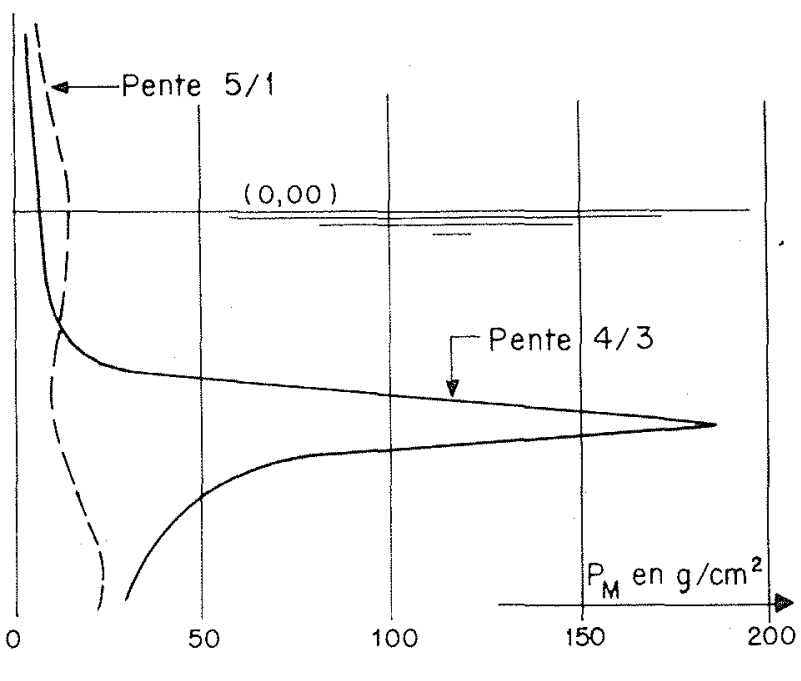

Fig. 6

deur infinie, car cette houle aurait déferlé sur les fonds avant d'atteindre l'ouvrage.

L'influence de la vitesse de reflux modifie l'épaisseur du matelas d'eau à l'arrivée de la vague suivante. Par aillenrs, pour les talus raides, l'énergie des houles courtes, concentrée, se dissipe sur une surface réduite, déterminant une pression plus grande que celle des houles longnes, alors que, pour les talus doux, le phénomène n'a plus le caractère de poinçonnement, et ce sont les houles lonsues, de retombée plus massive, qui déterminent les pressions les plus fortes.

Mais le sens du phénomène est à relenir pour intéressant, si même son retournement (de sens) n'est pas aussi accentué dans la réalité que le laisserait croire une enveloppe de cas discontinus. 
Les planches 15 et 16 du document 8.106 , reproduites (fig. 6), représentent la répartition des pressions le long du talus de la plaque, et cela pour deux inclinaisons extrêmes (4/3 et 10/2) de celui-ci, et les deux périodes adéquates :

$$
(\mathrm{T}=1,21 \text { et } 1,658)
$$

à la formation du déferlement sur lobstacle. Il saute aux yeux que, pour la combinaison déjà reconnue l'une des plus dangereuses, il $\mathrm{y}$ a une franche poussée de pression de $185 \mathrm{~g} / \mathrm{cm}^{2}$, un peu au-dessous du milieu de la couche d'eau; tandis que, sur le talus le plus doux, il y a comme deux maximums de pression, dont l'un est au niveau du repos, séparés par un minimum; ceci peut s'expliquer par la cassure du premier impact sous l'effet de la retombée relativement lente du jet de rive, et par le rebondissement du rouleau à une cote supérieure; le choc est plus réparti.

Pour les pentes douces, le facteur de la période est moins sensible que sur les pentes fortes; la profondeur: relative ou la longueur d'onde qui donne les pressions dangereuses est comprise dans un intervalle moins étroit.

\section{*}

Les planches 9 et 14 du document 8.106 (non reproduites ici) figurent, pour les divers talus, les courbes de pression, non plus en fonction de la profondeur relative $h / L$, mais d'après la période de la houle; celle-ci étant proportionnelle à la longueur, il n'est pas étonnant qu'on retrouve un peu les mêmes allures de courbes qu'aux planches 1 à 6 du document 8.106 (ou fig. 1 à 3 ), une poussée très brusque sur le talus le plus raide (planche 11), un étalement de la poussée, des courbes fluctuantes, avec influence plutôt défavorable des houles longues (jusqu'à un certain point) pour les talus doux, en opposition avec celle de houles courles, qui pointent pour une certaine période moyenne très précise et s'affaissent nettement pour les houles plus longues; la retombée des jets de rive a certainement un rôle dans ce phénomène, car on le voit aux expériences, en dépit de ce qu'on pent penser de la dispersion des résultats dans les parties aux fluctuations peu accentuées.

Pour chaque pente, il existe une profondeur relative ou une période critique particulièrement dangereuse au point de vue des pressions maximales, et cela, d'autant plus que la pente est plus raide, à cause de la vitesse du reflux de la vague sur la pente qui raidit le front du rouleau. Si le reflux est relativement lent, la frappe du roulcau est diminuée; il subsiste sous l'impact une lame d'eau non négligeable qui répartit la pression et fait cascader ou rehondir le rouleau dans un sens négatif, en remontant la pente). L'influence de la période sera donc moins sensible sur les pentes douces.

$$
\stackrel{*}{*}
$$

Les résultats précédents n'ont pas été considérablement modifiés avec des houles irrégulicres formees par le rattrapage et la composition de houles simples.

\section{Cas de la houle irrégulière.}

On se borne à reproduire une houle régulière équivalente, c'est-à-dire une houle dont la période et l'amplitude sont égales respectivement à la période moyenne et à l'amplitude significative de la houle irrégulière.

On s'est efforcé d'analyser l'aspect instantané de la gifle ou du ronleau déferlant sur le talus, de détecter ainsi les pressions maximales résu]tantes.

La différence à déceler entre les valeurs de pressions résultantes provient essentiellement de la fluctuation de la période, c'est-à-dire d'une fluctuation de la célérité. Les vagues de grande célérité rattrapent celles de célérité moindre et donnent lieu à des compositions d'amplitude. On peut obtenir ainsi des vagues d'amplitude supérieure à celle qui, en houle régulière équivalente, donnerait lieu à la cambrure-limite.

On a fait fluctuer les caractéristiques du variateur, périodiquement, entre des limites fixes.

On n'a opéré que sur deux pentes, la pente moyenne $2 / 1$ et la pente raide $4 / 3$.

On a fait fluctuer seulement : la période entre les deux extrêmes $1,6 \mathrm{~s}$ el $1,1 \mathrm{~s}$ d'une part, $1,3 \mathrm{~s}$ et $1,0 \mathrm{~s}$ d'autre part,

$\mathrm{T}_{1}-\mathrm{T}_{2}=$ soit $0,5 \mathrm{~s}$, soit $0,3 \mathrm{~s}$ en modèle.

Une autre caractéristique de la houle irrégulière est :

- La période moyenne $\mathrm{T}_{m}$ du secteur de variation de la période: $1,35 \mathrm{~s}$ et $1,15 \mathrm{~s}$, choisie au voisinage des périodes les plus défavorables;

- La période $\theta$ du cycle de la houle irrégulière, celle du retour du variateur de vitesse à la même position;

- L'excentricité $e$ du balteur qui permet d'obtenir les plus grandes cambrures juste avant l'ouvrage.

Les résultats sur houle régulière ayant montré que la pression maximale sur talus à $2 / 1$ était obtenue avec une période de $1,6 \mathrm{~s}$ (en modèle), c'est la valeur choisie pour la période moyenne $\mathrm{T}_{m}$; pour le talus $4 / 3, \mathrm{~T}_{m}=1,21 \mathrm{~s}$.

On a tâtonné par vingt-huit essais préliminaires avant de choisir la houle qui paraissait donner le maximum de pression. 
Il y a un peu de réflexion sur le talus, ce qui modifie l'amplitude significative.

Voir sur le document 8.106 les planches 17 et 19 (répartition des périodes en fréquences cumulées en $\%$ et périodes $\mathrm{T}_{1}$ de 1 à 1,5 ), les planches 18 et 20 ; amplitude maximale au voisinage de l'ourrage: millimetres en abscisses, pourcentages des fréquences en ordonnées.

Lamplitude significative du pseudo-clapotis sur la pente $2 / 1$ est de $185 \mathrm{~mm}$ sur modèle; celle de la houle régulière de période $1,6 \mathrm{~s}$ est de $200 \mathrm{~mm}$ environ.

Sur la pente $4 / 3$, les chilfres correspondants deviennent $90 \mathrm{~mm}$ et $110 \mathrm{~mm}$.

On voit que ces amplitudes sont très roisines; toutes les amplitudes supérieures sont dues à des rattrapages.

La répartition des pressions le long du talus est figurée sur les planches 21 et 22 du document 8.106 ; on y verra les pressions maximales en fonction de la cote où elles sont mesurées (pressions en g/ $\mathrm{cm}^{2}$ en abscisses; en ordonnées, les cotes en centimètres).

Pression maximale enregistrée sur le talus à 2/1:97 $\mathrm{g} / \mathrm{cm}^{2}$ ou, dans la nature, sous profondeur de $15 \mathrm{~m}$ (échelle $1 / 50$ ), $48 \mathrm{t} / \mathrm{m}^{2}$ et cela à $14 \mathrm{~cm}$, ou $7 \mathrm{~m}$ sous le niveau de repos; alors que la houle régnlière la plus dangereuse observée sur la pente $2 / 1$ était de $100 \mathrm{~g} / \mathrm{cm}^{2}$ ou $50 \mathrm{t} / \mathrm{m}^{2}$ à la cote $(-8,00)$. Les résultats sont de même ordre.

Si la période de $1,6 \mathrm{~s}$ a été la plus défavorable en houle régulière, ce ne sont pas les vagues correspondant à celte période qui sont les plus dangereuses en houle irrégulière.

Le rattrapage par une vague de période plus courte compose les vitesses des particules et les amplitudes. Il n'y a plus concentration de l'énergie en un point, les pressions dynamiques sont plus réparties.

La surcambrure favorise davantage les houles les plus courtes, et le maximum de pression serait donné plutôt par une vague correspondant sensiblement à la période de $1,4 \mathrm{~s}$.

Pour la pente $4 / 3$, les résultats sont $184 \mathrm{~g} / \mathrm{cm}^{2}$ el $14,3 \mathrm{~cm}$ sous le plan zéro, ou $92 \mathrm{t} / \mathrm{m}^{2}$ comme en houle régulière; la pression maximale se fait sentir à $(-7,15)$ et en houle régulière à $(-7,50)$. (planche 15 du document 8.106).

La comparaison entre les deux houles ne peut être complète.

On pourrait faire varier à l'infini les caractéristiques de la houle irrégulière, et on ne peut savoir si on reproduit la plus dangereuse, ce. qu'on a pu faire pour les houles régulières.

Conclusion provisome. - Les augmentations d'amplitude dues au rattrapage ne sont pas forcément un facteur d'augmentation de pression.

Cependant, il est probable qu'une houle irré- gulière, du fait des surcambrures provoquées, pourrait être plus dangereuse que la houle régulière équivalente, si celle-ci aborde l'ouvrage sans avoir atteint sa cambrure-limite.

\section{Seiches.}

Une séance a ćté consacrée à Baltimore à la question des seiches, sur lesquelles divers collègues américains ont fait des communications sur les recherches récentes en matières de seiches à Table Bay et au Cap en particulier, sur la dispersion des rapports entre la longueur et le creux des houles, ainsi que sur une étude de digues à talus soumise à la houle.

La discussion s'est ouverte sur les diverses causes des seiches; elle a abouti à la constatation qu'elles peuvent provenir soit d'une variation barométrique sensible (pour les lacs, c'est la seule), soit du déferlement des lames sur un rivage voisin, soit de ces deux causes simultanément pour les seiches marines, sans oublier l'effet amplificateur d'une résonance favorable.

Rien n'a été discuté de la longue note présentée par nous-même sur les divers modes d'amarrage des navires pour atténuer l'effet des seiches sur leur tenue.

\section{Travaux de M. Gille sur les efforts des lames.}

Deux notes résumant les travaux de M. Gruse, Ingénieur en chef de la Marine, ont été également présentées. Elles ont dommé lieu à des débats urès approfondis à la réunion du 30 novembre 1961 de la Commission d'Hydraulique fluviale et maritime de la S.H.F.

Cet auteur a attiré l'attention :

- Sur le degré de probabilité des hauteurs de houle, et non pas seulement sur les hauteurs d'elles-mêmes;

- Sur les modifications qu'apporte à la houle simple la superposition d'autres houles, donnant ainsi un mouvement complexe de plus grande amplitude;

- Sur les résultats d'observations qui permettent de déduire une relation entre la hauteur et la longueur de la houle.

Dans le cas de superpositions de houles, si on admet la théorie ordinaire du mouvement cycloïdal, il est facile de déduire les caractéristiques de la houle complexe résultante; ainsi apparaissent des houles dangereuses pour les navires, pouvant atteindre le double de la hauteur de la houle simple pour la même longueur, donc avec un escarpement bien plus accusé.

La probabilité des houles, déduite d'observations faites depuis vingt ans, permet de supputer 
les hauteurs maximales de houles simples et complexes, qui peuvent être rencontrées.

Il faut distinguer le cas des navires, où ces considérations sont valables pour l'action sur eux des houles, et le cas des ouvrages fixes, où le mouvement orbitaire par fonds inférieurs à la demi-longueur de la houle se transforme en un déferlement partiel ou total et dont la vitesse de propagation diminue.

Mais ce phénomène peut toucher aussi les navires s'engageant sur de petits fonds.

M. Gille parle égalcment d'un phénomène spécial de déferlement, mème en haute mer, quand le vent force et agit sur la partie haute des ondulations.

Il existe alors une masse échappant au mouvement orbitaire et accompagnant la houle avec la vitesse de propagation de celle-ci. La vitesse d'impact est bien plus élevée que celle des mouvements orbitaires.

M. Gille rappelle qu'une autre cause de déferlement est le creusement de la houle à l'approche des petits fonds devant un ouvrage fixe.

Le vent peut aussi intervenir en hâtant le déferlement.

M. Gille n'utilise que les formules classiques de la houle trochoïdale et des mouvements orbitaires sur fonds limités. Elles lui ont paru suffisantes comme représentation approximative de phénomènes complexes tels que la superposition d'ondes multiples.

Il s'est attaché, le 30 novembre, à expliquer l'incertitude traduite dans le rapport de M. Greco (Italie) par la forte difiérence existant entre un effort calculé et un effort enregistré. II est probable qu'il y a eu un phénomène de déferlement partiel qui explique cette différence.

Cette explication a été soumise à M. Greco, qui n'a pas encore répondu.

Le débat suivant l'exposé de M. Gille a été surtout animé par M. Mrche, de l'Entreprise des Grands Travaux Hydrauliques, dont nous retenons les aperçus hydrauliques suivants, en dehors de quelques considérations sur la consistance des digues verticales:

L'augmentalion de période qu'on peut constater sur une houle arrivant près du rivage par rapport à la houle mesurée au large, peut s'expliquer par le fait d'une composante de très longue période et de faible amplitude masquée par un éventail de houles plus courtes et plus cambrées; la composante de longue période subsiste seule au rivage, les autres s'estompent ou progressent plus lentement (allusion à la houle de période $19,3 \mathrm{~s}$ constatée au rivage de SaintJean-de-Luz, le 11 novembre 1960 , alors que la frégate météorologique n'a mesuré que des périodes de $15 \mathrm{~s}$ ).

Le doublement ou le triplement de l'amplitude moyenne peut se voir; mais, en pratique, il est noyé dans un plus grand nombre de composantes de vitesses et amplitudes diflérentes, en sorte qu'on n'apercoit qu'un brouillage très peu net que l'analyse spectrale seule permettrait de débrouiller, tout en dégageant la probabilité d'apparition de chaque onde.

L'énergie de la houle se traduit par une courbe très pointue quand il y a doublement d'amplitude par battement.

La façon d'évaluer la vitesse d'impact d'une lame déferlante sur un ouvrage vertical a donné lieu à un débat d'où aucune formule définitive n'a été retenue; mais à l'addition pure et simple de la vitesse de propagation de la houle (ou célérité) et de la vitesse orbitale propre des molécules du rouleau déferlant, a été opposée une sommation de termes affectés de certains coefficients de réduction (à propos de la divergence des constatations de M. Greco dans la théorie et la pratique).

Encore s'agit-il d'un effort local et non général susceptible d'affecter la tenue d'un ouvrage.

Quant à la faible augmentation de pression résultant d'une forte surélévation de la lame devant un ouvrage, antinomie constatée et mesurée, elle peut s'expliquer, selon M. Miche, par la faible épaisseur de la lame d'eau en jeu.

Des expériences sur modèle et de nouvelles observations dans la nature, peu coûteuses les unes et les autres, seraient les bienvenues pour ces divers points encore mal éclaircis.

L'accident de Gênes peut s'expliquer par des doublements de houle provoqués par des réflexions, ainsi que par des erreurs dans la constitution de l'ouvrage lors de ses coupures.

\section{Vœux.}

Quelques voux ont été exprimés à la suite des réunions de Baltimore.

Ils portaient sur le renforcement des organisations nationales poux l'étude des lames et des seiches; ce renforcement conduira à des mesures variées suivant les pays et leur organisation administrative.

Ils portaient également sur le recrutement de nouveanx membres, bien que la quantité des pays capables de s'intéresser sérieusement à de telles éludes soit fort linitée.

Ils portaient encore sur la restriction de la bibliographie aux seules pièces intéressant directement le Comité et l'Association des Congrès de Navigation.

En dernier lieu, le souhait a été exprimé que les sujets soumis à l'étude soient plus circonscrits, après les très belles théories, trop générales, mises en formules savantes et parfois contradictoires. 


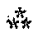

Nous avons visité le laboratoire de Washington. Nous arons vu une étude sur l'entretien d'une rivière à barre débouchant entre des jetćes, la résistance d'un parement de digue à talus constitué en «quadripodes » dans un très large canal, l'action comparée d'une même agi- tation sur une plage dégagée d'obstacles et sur une place barrée par un ouvrage vertical.

Enfin, des documents japonais et russes ont été remis au président du Comité. Ils portent sur les pressions dynamiques des vagues déferlant sur des brise-lames, sur les travaux poursuivis en U.R.S.S. en cette matière, notamment pour l'action sur les plages.

\section{I S CUS S I O N}

président: M. de Rouvilue:

M. le Président, après avoir exposé son rapport, ouvre la discussion.

M. Cailleex indique que l'influence favorable des pentes douces, justement soulignée par M. de Rouvinus pour les ouvrages en dur, s'étend aux plages sablenses naturelles; celles-ci ont une pente d'équilibre, dont on a intérêt à favoriser le maintien, par exemple par l'établissement ou de bon entretien d'épis.

D'autre part, le fait que la vague redescendante diminue l'effet de choe, ou la puissance de la vague montante suivante, se voit très bien en observant les galets, qui, dans ce cas, sont beatcoup moins déplacés.

Enfin, les recherches récentes confirment que l'indice d'émoussé des galets, dans la nature, est un bon enregistreur totalisateur des eftets produits par les houles sur les plages.

M. Ie Président donne, en exemple, les galets d'une exploitation de grès très durs au Val-André en Bretagne. Les galets étaient pointus au point origine des rejets de l'exploitation et arrondis deux ou trois cents mètres après.

M. PAAPE demande si on a étudié l'influence de la forme géométrique de la houle sur la valeur et la répartition des pressions.

M. Montaz répond que la forme géométrique de la houle dépend principalement des valeurs de la cambrure $\gamma$ et de la profondeur relative $h / L$. Dans les essais faits par M. Monraz, la houle était systematiquement reproduite en cambrure limite, $\gamma$ était fixé. On s'est done borné à étudier, pour chaque pente de talus, l'influence du paramètre $h / L$. Il ne semble pas possible de faire varier autre chose que l'amplitude et la profondeur relatives.

M. PAAPE pense que la position où la lame finale atteint la cambrure-limite dépend de la contribution des ondes élémentaires de différentes périodes qui composent cette lame. In présence d'une construction à paroi verticale, il est possible que la cambrure-limite d'une houle clémentaire ne soit jamais atteinte ou qu'elle le soit à une distance considérable de la construction. Si l'on a des lames a périodes différentes en superposition, la cambrure-limite peut être atteinte au front de l'ouviage.

A-t-on étudié successivement les périodes différentes qui sont nécessaires pour obtenir la condition la plus defavorable?

M. Monta répond que l'on n'a pas tellement exploré la houle irrégulière, mais qu'il serait intéressant de répontre à cette question.

M. le Plésident pense que M. Montaz et M. Panye pourraient rester en rapport pour approfondir la remarque de M. PAape.
M. Gille signale que, des photographies qui ont été projetées, il ressort qu'il y a deux phénomènes assez différents.

- la progression du déferlement qui se fait sans chutes; dans ce cas, les pressions peuvent être calculées facilement par les formules usuelles;

- la lame déferlante donnant une masse d'eau qui tombe normalement à la pente; dans ce cas, il y a effet de choc et pendant un temps très court, $1 / 100^{\circ}$ de seconde d'après les calculs sommaires de M. GiLles, une compression de l'eau qui introduit des pressions très fortes.

M. Gille pense qu'il y a lá une question intèressante a étudier : on dojt tenir compte de la compressibilité de l'eau pendant un temps très court. La force vive de la masse qui tombe se traduit en compression de la masse, qui devient immobile; puis l'eau est chassée. Ceia expliquerait ce phénomène anormal de très haute pression pour une pente déterminée. Pour les revêtements superficiels, il peut $y$ aroir un eftet de poinģonnement que M. le Président a signalé.

M. le Président dit qu'heuretusement, pour éviter la démolition des ouvrages, il $y$ a un facteur temps qui intervient.

M. Montaz dit que ces essais ont été continués par des enregistrements à très grande vitesse, ce qui permet d'évaluer le $1 / 500^{\circ}$ de seconde sur le phénomène de la gifle, particulièrement, et de montrer qu'après la sille if $y$ a un temps de relaxation. La fréquence de cette résonance après la gifle est de $1 / 100^{\circ}$ de seconde, ce qui aboutit au même résultat que celui de M. Gruxz.

On a vu, d'autre part, que la zone de haute pression est donnée par le point d'impact. Il semblerait aassi que la zone de haute pression soit donnée par la poche d'air enfermée par la lame d'eau.

M. Miche signale, au sujet de la question posée par M. Gille, que, lorsque des lames déferlent, il y a toujours une émulsion d'air dans l'eau. Du fait de la très faible compressibilité de l'eau pure, le coefficient moyen de compressibilité devient relativement beaucoup plus grand dès que $1 \%$, par exemple, du volume correspond $\dot{a}$ des bulles d'air. Ceci peut jouer un rôle dans le déroulement des phénomènes. En outre, il existe parfois des masses d'air emprisonnées sous la crête déferlante et comprimées lorsque ceile-ci bute sur un obstacle accore. Leur brusque détente pourrait expliquer la présence de gifles anormalement fortes constatées parfois, mais à titre sporadique.

D'une façon générale, le phénomène de la gifle sur les parois verticales a été étudié par M. Larras, sur modèle réduit, déjà en 1937. Son action est effectivement très brève, durée de l'ordre de $1 / 100^{\circ}$ de seconde; ceci fait présumer que la compressibilité des milieux soit liquides, soit solides, doit jouer un rôle appréciable. 
En ce qui concerne l'action d'ensemble d'une houle sur une digue verticale, il faut distinguer au moins deux cas :

Si l'ouvraye est soumis a une houle se trouvant assez Join du stade de déferlement, il se produit un clapotis plus ou moins parfait, pour lecquel la transmission horizontale d'énergie est théoriquement nulle en moyenne. Par conséquent, s'il n'y a pas déferlement, l'eau doit s'élever contre l'ouvrage, en principe deux fois plus haut que la houle incidente, pour absorber le flux initial d'énergie de la houle.

$\mathrm{Si}$, au contraire, la houle est très proche du déferlement avant d'arriver sur l'ourrage, le phénoméne est plus complexe. La totalité de l'énergie à ahsorber reste la même, mais on peut avoir localement des pressions beaucoup plus fortes. La structure doit donc être telle qu'elle puisse résister à des efforts concentrés. Dans l'ensemble, toutefois, il n'y a vaisemblablement pas de gros écarts quant au moment de renversement subi par. la digue. Ce moment devrait même ettre, en principe, un peu plus faible, car une partie du flux d'énergie est détruite par des phénomènes tourbillonnaires. Pour fixer ce point, il faudrait déterminer les pressions simultanées agissant en un assez grand nombre de niveaux de la paroi verticale, en faire l'intégrale, et ceci pour une gamme assez étendue de possibilités (houles de forme-limite ou franchement déferlantes - variation de la longueur relative de ces houles, c'est-à-dire du rapport de la longueur d'onde et de la profondeur au repos, etc.).

Lorsqu'il n'y a pas déferlement de la houle incidente ni brisure du clapotis résultant, la courbe des pressions

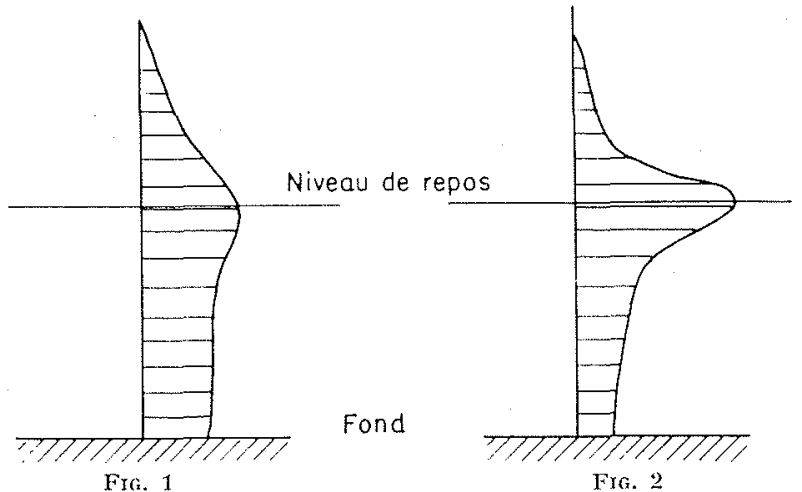

classique ressortant de nombreux essais a la forme suivante (fig. 1).

Si le phénomène du déferlement intervient, la courbe figure 1 est plus ou moins modifiée avec une forte pointe de pression (fig. 2); donc, il serait indiqué de pouvoir fixer la position et l'intensité par des essais systématiques sur modèle réduit, par exemple.

Pour la raison exposée ci-dessus, il est probable cependant que la surface de ce diagramme n'est pas plus grande que dans le cas 1 et même un peu inférieure, ainsi que le moment de renversement produit sur l'ouvrage.

M. le Président clot la discussion, plus personne $\mathrm{n}$ demandant la parole.

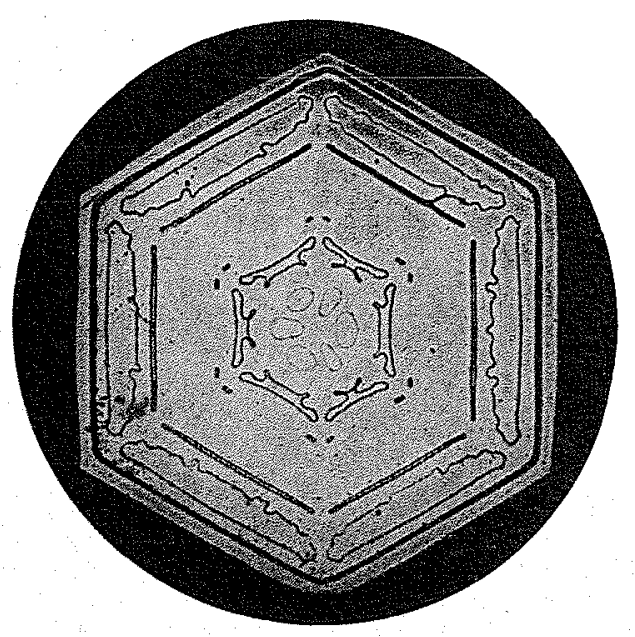

